\title{
Prática da automedicação entre acadêmicos do curso de enfermagem de uma instituição de ensino superior
}

Practice of self-medication among academic nursing students of a higher education institution

T. S. Santos ${ }^{1}$; M. M. Almeida ${ }^{1}$; É. V. M. Pessoa ${ }^{2}$ N N. M. Pessoa² ${ }^{2}$ H. D. S. Siqueira $^{3}$; J. M. N. Silva ${ }^{4}$; R. N. C. M. Junior ${ }^{4}$; A. C. E. Rodrigues ${ }^{4}$; F. L. Silva A. B. S. Silva ${ }^{5}$; G. T. Pessoa ; F. C. A. Sousa

\author{
${ }^{1}$ Faculdade de Tecnologia e Educação Superior e Profissional, 64001-120, Teresina - PI, Brasil \\ ${ }^{4}$ Faculdade de Ciências e Tecnologia do Maranhão, 65606-020, Caxias - MA, Brasil \\ ${ }^{5}$ Centro de Ciências Agrárias, Universidade Federal do Piauí, 64049550, Teresina - PI, Brasil \\ ${ }^{6}$ Faculdade de Ciências Médicas, Universidade Estadual do Piauí, 64001280, Teresina - PI, Brasil \\ *andrezzab1@hotmail.com \\ (Recebido em 23 de março de 2018; aceito em 31 de julho de 2018)
}

${ }^{2}$ Curso de Nutrição, Faculdade de Ciências e Tecnologia do Maranhão, 65606-020, Caxias - MA, Brasil

${ }^{3}$ Curso de Farmácia, Faculdade de Ciências e Tecnologia do Maranhão, 65606-020, Caxias - MA, Brasil

\begin{abstract}
A automedicação é uma atividade preocupante no país e conta com uma prática abusiva no intuito de aliviar sintomas de imediato sem consentimento médico. O objetivo desse estudo foi caracterizar a prática da automedicação entre os acadêmicos de Enfermagem de uma Instituição de Ensino Superior. Trata-se de uma pesquisa de campo, do tipo descritivo, com abordagem quantitativa, por meio da aplicação de um questionário abordando o comportamento dos acadêmicos de enfermagem acerca da prática da automedicação. Utilizou para análise dos dados o software estatístico SPSS 18.0. Quanto à realização da prática da automedicação $91,2 \%$ afirmaram realizar e $8,7 \%$ não realizam. Em relação à frequência da prática, $55,7 \%$ realizam-na frequentemente. O principal motivo da automedicação é a dificuldade e demora no atendimento médico $(38,46 \%)$, sendo que $60,7 \%$ afirmam ter conhecimento dos efeitos colaterais e contraindicações. Os medicamentos mais utilizados foram analgésicos e anti-inflamatórios. Portanto, fazse necessário a criação de estratégias voltadas para a conscientização dessa parcela da população, visando à redução desta prática entre eles.

Palavras chaves: Automedicação, Estudantes de Enfermagem, Instituições Acadêmicas.
\end{abstract}

Self-medication is a worrying activity in the country and has an abusive practice in order to alleviate symptoms immediately without medical consent. The aim of this study was to characterize the practice of self-medication among Nursing students of a Higher Education Institution. It is a field research, of the descriptive type, with a quantitative approach, through the application of a questionnaire addressing the behavior of nursing students about the practice of self-medication. The SPSS 18.0 statistical software was used for data analysis. Regarding the practice of self-medication, 91.3\% said they performed and $8.8 \%$ did not. Regarding the frequency of practice, $55.7 \%$ performed it frequently. The main reason for selfmedication is difficulty and delay in medical care $(38.46 \%)$, and $60.7 \%$ say they are aware of the side effects and contraindications. The most commonly used drugs were analgesics and anti-inflammatories. Therefore, it is necessary to create strategies aimed at raising awareness of this part of the population, aiming at reducing this practice among them.

Keywords: Self-medication, Nursing Students, Academic Institutions.

\section{INTRODUÇÃO}

A prática da automedicação vem se tornando cada vez mais uma problemática para os serviços de saúde, não somente no Brasil, mas também em outros países. Essa prática consiste no consumo de medicamentos com o objetivo de tratar ou aliviar sintomas ou doenças, ou mesmo de promover a saúde, independente da orientação/prescrição de um profissional [1]. Os medicamentos apresentam essencialmente certo valor simbólico, transcendendo a sua atividade terapêutica, tornando assim um forte fator justificável para o seu consumo e seu uso irracional [2].

Os problemas de saúde justificam a necessidade real da utilização de medicamentos, entretanto essa prática da automedicação também é motivada por fatores culturais e comportamentais [3]. Existem alguns motivos que favorece o indivíduo a se automedicar, onde a maioria relata ter 
dificuldade para conseguir consulta médica e queixam-se do alto custo em serviços particulares de saúde, como problemas relacionados à falta de regulamentação e fiscalização daqueles que administram o medicamento também favorece essa prática [4].

Corroborando com essa ideia, Damasceno et al. (2007) [4] relata que essa prática causa complicações até mais graves ao comparada com o problema na qual o indivíduo buscou solucionar ao se automedicar; como exemplo: mascaramento de doenças evolutivas, atraso em diagnósticos e tratamentos médico, e problemas financeiros, uma vez que esta prática gera aumento dos custos com a saúde.

Atualmente, todos os medicamentos estão inseridos aos serviços de atenção à saúde. Devido a essa ampla inserção, sua utilização inadequada passou a ser um sério problema de saúde pública, uma vez que o uso indevido ocasiona em problemas de saúde o que faz com os indivíduos busquem atendimentos gerando assim mais gastos públicos [5].

De acordo com Rios et al. (2013) [6], são inúmeros os estudos que apresentam a alta prevalência da prática da automedicação entre acadêmicos de diferentes cursos da área da saúde; acadêmicos estes que serão os futuros responsáveis por conscientizar a sociedade sobre os problemas relacionados com a automedicação. $\mathrm{O}$ objetivo desse estudo foi caracterizar a prática da automedicação entre os acadêmicos de Enfermagem de uma Instituição de Ensino Superior.

\section{METODOLOGIA}

O trabalho encontra-se com aprovação do comitê de ética em pesquisa (CEP) pela Plataforma Brasil sob o parecer $\mathrm{n}^{\circ}$ 2.043.824. Trata-se de um estudo descritivo, transversal, com abordagem quantitativa. A pesquisa foi realizada na Faculdade de Ciências e Tecnologia Maranhão FACEMA, no período de março a novembro de 2015. Os participantes da pesquisa foram os acadêmicos do curso de Enfermagem que aceitaram responder ao instrumento de coleta de dados; sendo que a Faculdade possui 631 alunos de Enfermagem distribuídos em 14 turmas, em 2 turnos.

A amostra da pesquisa foi composta por 240 alunos, porém do tipo estratificada, sendo 30 alunos por cada período, do $1^{\circ}$ ao $8^{\circ}$. A amostra da pesquisa foi feita com base em um cálculo de amostragem para população finita, com grau de confiança de $95 \%$ e margem de erro de $5 \%$.

A coleta de dados ocorreu no período de outubro a novembro de 2015 mediante aplicação de um questionário contendo questões pré-elaboradas sobre o tema automedicação, além de questões que traçam o perfil socioeconômico dos participantes, somando um total de 07 questões fechadas (APÊNDICE B), aplicado a 30 acadêmicos do curso de enfermagem de cada período de acordo com o cálculo amostral e de acordo com os critérios de inclusão: ser maior de 18 anos; está devidamente matriculado na instituição e ser do curso de enfermagem. Os estudantes que aceitaram participar formalmente na pesquisa assinaram o Termo de Consentimento Livre e Esclarecido.

Os dados foram organizados e tabulados utilizando o Microsoft Excel ${ }^{\circledR}$ versão 2010 para Windows e as análises estatísticas foram feitas por meio do SPSS ${ }^{\circledR}$ versão 18.0 para Windows (SPSS Inc. Chicago, IL 60606, EUA). Os dados foram apresentados por meio de frequência simples e absoluta. Para verificar associação entre a prática de automedicação e as variáveis qualitativas foi utilizado o teste Qui-quadrado de Pearson $\left(\chi^{2}\right)$ considerando em todas as análises realizadas um nível de significância de 5\%.

\section{RESULTADOS}

\subsection{Caracterização sociodemográfica dos participantes da pesquisa}

Quanto à faixa etária, o estudo revelou que a maior prevalência foi de acadêmicos entre 21-30 anos (67,1\%). Quanto ao estado civil, a maioria (76,3\%) eram solteiros, e 17,5\% casados. Em relação à raça/cor o estudo revelou que a cor predominante é a parda (62,9\%); sendo a branca de $18,3 \%$. Com base na renda familiar, o estudo revelou que a maioria dos acadêmicos apresenta renda familiar entre 1 a 2 salários mínimos $(56,7 \%)$ e que $29,2 \%$ recebem entre 3 a 5 salários mínimos, conforme a Tabela 1. 
Tabela 1. Caracterização sociodemográfica dos participantes da pesquisa. Caxias - MA, 2015. (n=240). Fonte: Pesquisa Direta, 2015. SM: salário mínimo.

\begin{tabular}{|c|c|c|}
\hline Variáveis & $\mathbf{n}$ & $\%$ \\
\hline \multicolumn{3}{|l|}{ Sexo } \\
\hline Masculino & 50 & 20,8 \\
\hline Feminino & 190 & 79,2 \\
\hline \multicolumn{3}{|l|}{ Faixa etária } \\
\hline Menor 20 anos & 52 & 21,7 \\
\hline 21-30 anos & 161 & 67,1 \\
\hline 31-40 anos & 21 & 8,8 \\
\hline Maior 40 anos & 06 & 2,5 \\
\hline \multicolumn{3}{|l|}{ Estado civil } \\
\hline Solteiro $(a)$ & 183 & 76,3 \\
\hline Casado (a) & 42 & 17,5 \\
\hline Divorciado (a) & 07 & 2,9 \\
\hline Outros arranjos & 08 & 3,3 \\
\hline \multicolumn{3}{|l|}{ Raça/Cor } \\
\hline $\operatorname{Branco}(a)$ & 44 & 18,3 \\
\hline $\operatorname{Negro}(a)$ & 42 & 17,5 \\
\hline Pardo (a) & 151 & 62,9 \\
\hline Amarelo (a) & 03 & 1,3 \\
\hline \multicolumn{3}{|l|}{ Renda familiar } \\
\hline Menor 1 SM & 27 & 11,3 \\
\hline 1 a $2 S M$ & 136 & 56,7 \\
\hline 3 a $5 S M$ & 70 & 29,2 \\
\hline$\geq 5 S M$ & 07 & 2,9 \\
\hline Total & 240 & 100,0 \\
\hline
\end{tabular}

Em relação à prática da automedicação, a pesquisa revelou que de uma amostra de 240 acadêmicos, 91,3\% (IC95\%: 87,6-94,8) afirmaram realizar automedicação e apenas 8,8\% (IC95\%: 0,5 - 12) afirmaram não realizar essa prática (Tabela 2).

Tabela 2. Prevalência de automedicação entre os participantes da pesquisa. Caxias - MA, 2015. n=240). Fonte: Pesquisa Direta, 2015; IC95\%: Intervalo de confiança de $95 \%$.

\begin{tabular}{cccc}
\hline Automedicação & $\boldsymbol{n}$ & $\boldsymbol{\%}$ & $\boldsymbol{I C 9 5 \%}$ \\
\hline Sim & 219 & 91,2 & $87,6-94,8$ \\
Não & 21 & 8,7 & $0,5-12,3$ \\
\hline Total & $\mathbf{2 4 0}$ & $\mathbf{1 0 0 , 0}$ & - \\
\hline
\end{tabular}

Com relação à frequência com que o participante pratica a automedicação, apresenta os principais motivos que justifique a prática e apresenta o conhecimento dos acadêmicos frente aos efeitos colaterais e contraindicações dos medicamentos utilizados por eles na prática da automedicação; sendo que a amostra é composta apenas por aqueles que afirmaram se automedicar $(\mathrm{n}=221)$. Com base na análise da Tabela 3 , quanto a primeira variável sobre a frequência com que os participantes se automedicavam, observou-se que $123(55,7 \%)$ dos praticantes se automedicam com frequência; outros $50(22,6 \%)$ referem fazer uso de medicamento de maneira mais frequente e apenas $48(21,7 \%)$ referem fazer uso raramente (Tabela 3$)$. 
Tabela 3. Dados relativos à prática da automedicação pelos participantes da pesquisa. Caxias - MA, 2015. (n=221). Fonte: Pesquisa Direta, 2015.

\begin{tabular}{|c|c|c|}
\hline Variáveis & $n$ & $\%$ \\
\hline \multicolumn{3}{|l|}{ Frequência da prática de automedicação } \\
\hline Frequentemente & 50 & 22,6 \\
\hline 1 vez ao mês & 123 & 55,7 \\
\hline Raramente & 48 & 21,7 \\
\hline \multicolumn{3}{|l|}{ Motivo da prática da automedicação } \\
\hline Dificuldade ou demora no atendimento médico & 85 & 38,46 \\
\hline Facilidade em adquirir o medicamento no balcão da farmácia & 44 & 19,9 \\
\hline Pelo fato de muitos utilizarem na prática da automedicação & 12 & 5,42 \\
\hline Achar-se esclarecido por ser acadêmico de enfermagem & 04 & 1,9 \\
\hline Por já ter tomado o mesmo medicamento em outras ocasiões & 75 & 33,9 \\
\hline Outros & 01 & 0,44 \\
\hline \multicolumn{3}{|l|}{$\begin{array}{l}\text { Conhecimentos dos efeitos colaterais e contraindicação dos } \\
\text { medicamentos utilizados }\end{array}$} \\
\hline $\operatorname{Sim}$ & 134 & 60,7 \\
\hline Não & 87 & 39,3 \\
\hline Total & 221 & 100,0 \\
\hline
\end{tabular}

A Tabela 4 trata dos medicamentos utilizados na prática da automedicação pelos acadêmicos de Enfermagem. De acordo com a análise desta tabela, a maioria faz uso do paracetamol (75,9\%); sendo que 67,6\% utiliza dipirona; $55,7 \%$ utiliza diclofenaco; 55,3\% faz uso de vitamina C e $53 \%$ faz uso de amoxicilina.

A figura 1 apresenta o conhecimento dos acadêmicos quanto à crença de que a automedicação pode causar danos à saúde. O estudo mostrou que $92,1 \%$ afirmam que essa prática pode ocasionar em danos à saúde, sendo que apenas $7,9 \%$ não acredita que essa prática pode ser prejudicial.

3.2 Avaliações da prática da automedicação e sua relação com as variáveis sociodemográficas e a variável quanto à crença em danos à saúde

Quanto à associação de variáveis, a pesquisa utilizou para essa análise o teste de quiquadrado de Pearson, através da qual foi avaliada a associação da prática da automedicação com a caracterização sociodemográfica dos participantes. Os resultados revelam que o sexo masculino associou-se positivamente $(\mathrm{p}=0,041)$. Quanto às demais variáveis não obteve significância positiva (Tabela 5). 
Tabela 4. Distribuição dos medicamentos utilizados pelos participantes na prática da automedicação. Caxias-MA, 2015. (n=221). Fonte: Pesquisa Direta, 2015. * Os participantes poderiam optar por um ou mais medicamentos.

\begin{tabular}{rcc}
\hline Medicamentos & $\boldsymbol{N}$ & $\mathbf{\%}$ \\
\hline Paracetamol & 166 & 75,9 \\
Dipirona & 148 & 67,6 \\
Nimesulida & 145 & 66,2 \\
Diclofenaco Potássio & 126 & 55,7 \\
Vitamina C & 121 & 55,3 \\
Amoxicilina & 116 & 53,0 \\
Complexo B & 75 & 34,2 \\
Albendazol & 65 & 29,7 \\
Mebendazol & 60 & 27,4 \\
Escopolamina & 59 & 26,9 \\
Metronidazol & 50 & 20,8 \\
Secnidazol & 39 & 17,8 \\
Azitromicina & 31 & 14,2 \\
Ciprofloxacino & 25 & 11,4 \\
Ampicilina & 20 & 9,1 \\
Bromoprida & 19 & 8,7 \\
Dimenidrinato & 11 & 5,0 \\
Metoclopramida & 07 & 3,2 \\
Diazepam & 05 & 2,3 \\
Clonazepam & 01 & 0,5 \\
Flurazepam & 01 & 0,5 \\
Ibuprofeno & 01 & 0,5 \\
Ondansetrona & 01 & 0,5 \\
& 01 & 0,5 \\
\hline
\end{tabular}

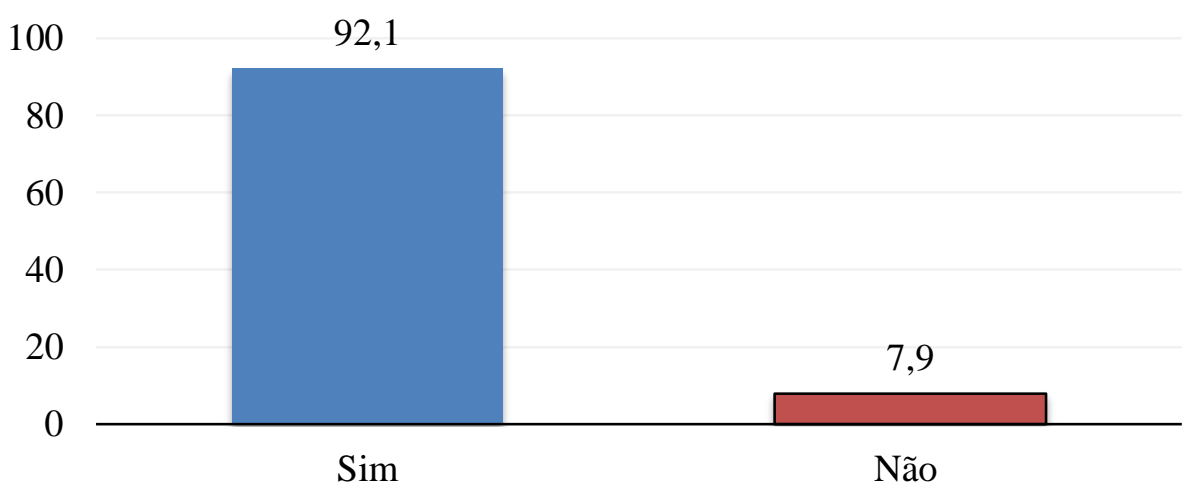

Figura 1. Distribuição das respostas referentes à crença em danos causados pela prática da automedicação. Caxias - MA, 2015. Fonte: Pesquisa Direta, 2015 
Tabela 5. Prevalência de automedicação entre os participantes segundo a caracterização sociodemográfica e danos à saúde por parte da automedicação. Caxias - MA, 2015. Fonte: Pesquisa Direta, 2015. *Teste Qui-quadrado de Pearson

\begin{tabular}{|c|c|c|c|c|c|}
\hline & \multicolumn{4}{|c|}{ Automedicação } & \multirow[b]{2}{*}{$\boldsymbol{P}$} \\
\hline & \multicolumn{2}{|c|}{ Sim } & \multicolumn{2}{|r|}{ Não } & \\
\hline & n & $\%$ & n & $\%$ & \\
\hline Sexo & & & & & 0,041 \\
\hline Masculino & 42 & 84,0 & 08 & 16,0 & \\
\hline Feminino & 177 & 93,2 & 13 & 6,8 & \\
\hline Faixa etária & & & & & 0,596 \\
\hline Menor 20 anos & 46 & 88,5 & 06 & 11,5 & \\
\hline 21-30 anos & 149 & 92,5 & 12 & 7,5 & \\
\hline Maior 30 anos & 24 & 88,9 & 03 & 11,1 & \\
\hline Estado civil & & & & & 0,845 \\
\hline Casado (a) & 181 & 91,4 & 17 & 8,6 & \\
\hline Não casado (a) & 38 & 90,5 & 04 & 9,5 & \\
\hline Raça/Cor & & & & & 0,616 \\
\hline $\operatorname{Branco}(a)$ & 41 & 93,2 & 03 & 6,8 & \\
\hline Não branco $(a)$ & 178 & 90,8 & 18 & 9,2 & \\
\hline Renda familiar & & & & & 0,718 \\
\hline Menor 2 SM & 148 & 90,8 & 15 & 9,2 & \\
\hline Maior 2 SM & 71 & 92,2 & 06 & 7,8 & \\
\hline $\begin{array}{l}\text { Automedicação } \\
\text { acarreta danos à saúde }\end{array}$ & & & & & 0,775 \\
\hline Sim & 202 & 91,4 & 19 & 8,6 & \\
\hline Não & 17 & 89,5 & 02 & 10,5 & \\
\hline
\end{tabular}

\section{DISCUSSÃO}

De acordo com um estudo realizado por Souza et al. (2011) [7], o alto índice da automedicação em curso de enfermagem é observada principalmente em mulheres, onde tal explicação está no fato de que os cursos da área da saúde em sua maior parte são compostos por pessoas do sexo feminino.

Sobre a faixa etária, o estudo revelou que a maioria dos participantes está na faixa etária de 21 a 30 anos de idade, algo bastante comum, pois muitos conseguem adentrar no curso superior com essa idade, logo após sua formação no ensino médio [1]. Alguns estudos revelam que o consumo de medicamentos costuma aumentar à medida que as pessoas envelhecem, uma vez que este fato é justificável devido ao aparecimento de sintomas comuns que ocorrem no período de envelhecimento. Entretanto, nos últimos anos a realização da automedicação tem ocorrido mais entre as pessoas mais jovens, tal fato deve-se a maneira de como os jovens estão cada vez mais preocupados com a saúde e logo vão buscar uma maneira de tratar ou prevenir doenças [8]. Uma pesquisa semelhante feita por Fontanella et al. (2013) [9] apresentou que a média da idade dos indivíduos que praticam a automedicação é de 25 anos.

O presente estudo também avaliou a situação conjugal e renda familiar dos participantes, na qual o resultado mostrou que a maioria dos participantes entrevistados são solteiros e possuem uma renda de 1 a 2 salários mínimos. Um estudo realizado por Vitor et al. (2008) [10] com pessoas residentes na cidade de Porto Alegre, RS, verificou que mais da metade das pessoas que realizam a automedicação são solteiras, sendo que nesse mesmo estudo viu-se que os mesmos possuíam uma renda de 1 a 3 salários, e o fato deles possuírem uma acima da média salarial, consideram ter mais facilidade de adquirir um medicamento sem a necessidade de uma consulta médica, assim podem tratar-se ou prevenir-se de qualquer doença facilmente.

No que se refere à prática da automedicação, o estudo apresentou a taxa de indivíduos que afirmava ou não se automedicar, onde de acordo com os resultados obtidos, 91,2\% dos 
acadêmicos de enfermagem se automedicam e apenas $8,7 \%$ relataram não praticar a automedicação. Um estudo semelhante a este foi realizado por Silva e Rodrigues (2014) [11], no qual se buscou a prevalência da automedicação com estudantes da área da saúde, para tal estudo, tendo sido encontrado que maioria dos entrevistados afirma fazer uso de medicamentos sem prescrição médica.

Em relação aos estudantes universitários, especialmente os da área da saúde, estudos mostram que a automedicação se apresenta de maneira elevada e que ocorre de maneira frequente. Tal atitude está relacionada a diversos fatores, sendo um deles a autoconfiança gerada pelo maior conhecimento teórico e prático pela sua formação [12]. Outro dado relevante neste estudo é a frequência com que os acadêmicos consomem medicamentos, na qual ao investigar esse fator, os acadêmicos referiram fazer uso de vez em quando de algum medicamento e outros referiram utilizar de maneira mais frequente, sendo que apenas uma pequena parte usa de maneira mais rara. Dessa forma o resultado demonstra como a automedicação é uma prática rotineira, que vem sendo cada vez mais praticada, sem qualquer tipo de preocupação por parte daqueles que realizam.

Ao avaliar a prática da automedicação em uma instituição acadêmica de Recife- PE, onde verificou-se que os participantes fizeram uso de medicamentos nos últimos quinze dias que antecederam à entrevista da pesquisa esta, por sua vez, apresentou que 65,5\% dos entrevistados confirmaram ter feito uso de algum medicamento [13].

$\mathrm{O}$ autocuidado realizado por meio da automedicação é bastante comum e é realizado por inúmeros motivos. O presente estudo mostrou alguns motivos, sendo que três motivos se apresentaram com mais frequência. Dentre os principais motivos de se realizar a automedicação, $39,54 \%$ afirmaram que já tem conhecimento, pois já haviam "usado o mesmo medicamento em outras ocasiões". O segundo motivo relatado pelos acadêmicos entrevistados é a dificuldade ou demora no atendimento médico na rede pública, e o terceiro principal motivo seria a facilidade em adquirir o medicamento no balcão da farmácia. Em um estudo semelhante a este, uma pesquisa apresentou alguns dos motivos que levam as pessoas a se automedicar, sendo que três motivos tiveram maior destaque, na seguinte ordem: dificuldade de acesso aos serviços de saúde, facilidade de acesso a fármacos e experiência com o fármaco [14].

No que concerne ao conhecimento dos participantes em relação aos efeitos adversos e contraindicações dos medicamentos, a pesquisa mostrou que 60,7\% dos acadêmicos que se automedicavam possuíam conhecimento dos efeitos adversos e as contraindicações e 39,3\% referiram se automedicar sem ter conhecimentos dos efeitos adversos sobre o organismo. A pesquisa feita por Ribeiro et al. (2010) [15] com graduandos de uma Instituição de Nível Superior revelou que os acadêmicos de cursos da área da saúde conhecem mais os efeitos adversos e contraindicações dos medicamentos do que aqueles que estudam em outras áreas de ensino.

No que diz respeito aos tipos de medicamentos mais utilizados por esses acadêmicos, conforme a tabela 4, o estudo revelou o paracetamol, dipirona, nimesulida, diclofenaco, vitamina $\mathrm{C}$ e amoxicilina como sendo os mais consumidos. Em um estudo semelhante a este realizado por Jesus et al. (2012) [16] com estudantes da área da saúde, mostrou que a dipirona sódica e paracetamol eram as drogas da classe dos analgésicos mais consumidos. Tal explicação está relacionada às principais queixas que os estudantes afirmam ter como, por exemplo: dores de cabeça, dores músculo- esqueléticas e cólicas menstruais.

A dipirona e o paracetamol são os analgésicos mais prescritos no Brasil, e também os mais utilizados na prática da automedicação, pois são facilmente adquiridos em farmácias e em comércios [14]. Estudos sobre a automedicação em outros países mostram que a dipirona tem seu uso restrito nos EUA e em vários países da Europa, uma vez que há pesquisas que comprovam que este medicamento está relacionado às causas de problemas como agranulocitose, complicações gastrointestinais, anemia aplástica e anafilaxia [18].

Segundo Silva e Lourenço (2014) [11], diariamente mais de 30 milhões de pessoas ingerem anti-inflamatórios não esteroidais, (AINES) sem nenhum tipo de prescrição médica, dentre eles: ibuprofeno, diclofenaco, meloxicam, e nimesulida. Dentre esses citados um dos mais comuns é a nimesulida, cuja a maior parte das pessoas que usa algum anti-inflamatório na prática da automedicação refere que esse medicamento é o de primeira escolha. De acordo com Silva e Lourenço (2014) [11], medicamentos como estes são facilmente adquiridos e estão à venda nas prateleiras das farmácias e comércios, sem a necessidade da prescrição médica. 
A nimesulida é um fármaco anti-inflamatório que atua no controle de problemas de inflamação aguda ou crônica e no tratamento de processos inflamatórios pós-cirúrgicos. Entretanto, há estudos que mostram que esse medicamento apresenta uma alta toxicidade quando usado de maneira indiscriminada, estando ligado a problemas como a toxicidade de células hepáticas, problemas gastrintestinais e toxicidade renal [19].

O paracetamol (acetaminofeno) é um fármaco que possui propriedades analgésicas (alivia a dor) e propriedades antipiréticas eliminando ou prevenindo a febre em adultos e crianças, diminuindo a temperatura corporal quando esta for superior a $37^{\circ} \mathrm{C}$. É um dos fármacos mais vendidos em todo o mundo, estando disponível no mercado na forma de genérico e sob vários nomes comerciais entre os quais o Ben-u-ron, Panadol, Tylenol, Supofen, Pantadolor, o Panasorbe e o Efferalgan [20]

Com relação ao medicamento da classe dos antimicrobianos mais utilizados, $33 \%$ dos indivíduos entrevistados afirmaram fazer uso da amoxicilina, enquanto $10 \%$ utilizam a cefalexina ou azitromicina [21]. Embora todos os antimicrobianos devessem ser liberados para venda mediante apresentação de prescrição médica, infelizmente são vendidos livremente e são comumente escolhidos para a prática da automedicação.

De acordo com dados do Ministério da Saúde (2009), uma das maiores causas de resistência bacteriana está relacionada ao uso indiscriminado de antimicrobianos, sendo que esses dados estão sendo usados para classificá-los como um dos medicamentos que mais provocam riscos à saúde dos pacientes, em comparação aos entorpecentes e psicotrópicos [22].

$\mathrm{Na}$ análise onde se verificou a associação da prática da automedicação com a caracterização sociodemográfica dos participantes, obteve-se associação positiva com o sexo, no qual evidencializou que mulheres realizam a automedicação mais do que homens, deve-se para isso a justificativa de que as mulheres são mais frágeis que os homens e cuidam-se mais de si mesma, além de que procuram evitar problemas de saúde por preocuparem-se com sua aparência estética e seu corpo.

\section{CONSIDERAÇÕES FINAIS}

A automedicação é uma prática que vem tornando-se bastante comum em vários centros de ensino superior, e isso vem ganhando grande destaque em pesquisas, uma vez que essa prática tem apresentando-se elevada, sobretudo entre acadêmicos de cursos da área da saúde. Fatores como o nível de conhecimento, autoconfiança gerada pela formação acadêmica, condição financeira e precariedade dos serviços de saúde são alguns dos motivos citados em estudos que interfere na vida do acadêmico, durante a tomada de decisão para uso de medicamentos por orientação própria.

No que diz respeito à área da saúde, existe hoje uma grande preocupação no que se refere à formação de novos enfermeiros para o mercado de trabalho. Conhecer a realidade da automedicação realizada pelos acadêmicos poderá contribuir para que instituições de ensino de enfermagem programem estratégias de cunho educativo, produzindo assim um melhor conhecimento e um maior incentivo aos acadêmicos quanto aos efeitos nocivos associados à prática da automedicação.

\section{REFERÊNCIAS}

1. Rosse WJD, Mouro VGS, Carvalho CA. Perfil da automedicação em acadêmicos do curso de farmácia da Univiçosa, Viçosa- MG. Rev Bras Farm. 2011;92(3):186-190.

2. Silva IM, Catrib AMF, Matos VC, Gondim APS. Automedicação na adolescência: um desafio para a educação em saúde. Rev Cienc Saude Colet. 2011;16(1):1651-1660.

3. Lyra Junior PD, Neves AS, Cerqueira KS, Marcellini PS, Marques TC, Barros JAC. Influência da propaganda na utilização de medicamentos em um grupo de idosos atendidos em uma unidade básica de saúde em Aracaju (SE, Brasil). Rev Cienc Saude Colet. 2010;15(3):3497-3505.

4. Damasceno DD, Terra FDS, Zanetti HHV, D'Andréa ÉD, Silva HLRD, Leite A. Automedicação entre graduandos de enfermagem, farmácia e odontologia da Universidade Federal de Alfenas. Rev Min Enfermag 2007;11(1):48-52, doi:S1415-27622007000100008. 
5. Costa CMFN, Silveira MR, Assis Acurcio F, Junior AAG, Guibu IA, Costa KS, Nascimento RCRM. Utilização de medicamento pelos usuários da atenção primária do Sistema Único de Saúde. Rev Saúde Pública. 2017;1(1):1-11.

6. Rios MF, Souza WA, Souza Siqueira VM, Podesta MHMC, Zuba AG, Machado JCFS, Ferreira EB. Perfil da automedicação dos alunos de uma escola técnica do Sul de Minas Gerais. Rev UninCor. 2013;11(2):420-421, doi:10.5892/ruvrd. v11i2. 420431.

7. Souza LAF, Silva CD, Ferraz GC, Sousa FAEF, Pereira LV. Prevalência e caracterização da prática de automedicação para alívio da dor entre estudantes universitários de enfermagem. Rev Lat Americ Enferm. 2011;19(2).

8. Junior JG, Moura SEDS, Dantas GCL, Lima AM, Brito WSB, Siebra BDOB, Cândido EL. Influência da publicidade na automedicação na população de um município brasileiro de médio porte. J Health \& Biol Sc, 2018;6(2):152-155.

9. Fontanella FG, Galato D, Remor KVT. Perfil de automedicação em universitários dos cursos da área da saúde em uma instituição de ensino superior do sul do Brasil. Rev Bras Farm. 2013;94(2):154-60.

10. Vitor RS, Lopes CP, Menezes HS, Kerkhoff CE. Padrão de consumo de medicamentos sem prescrição médica na cidade de Porto Alegre, RS. Cienc Saúde Coletiva. 2008;13:737-743, doi:10.1590/S141381232008000700024.

11. Silva LAF, Rodrigues AMS. Automedicação entre estudantes de cursos da área de saúde. Rev Bras Farm. 2014;95(3):961-975.

12. Silva RC, Oliveira TM, Casimiro TS, Vieira KA, Tardivo MT, Junior MF, Restini CB. Automedicação em acadêmicos do curso de medicina. Rev Med-Rib Preto. 2012;45(1):5-11, doi:10.11606/issn.21767262.v45ilp5-11.

13. Aquino DS, Barros JAC, Silva MDP. A automedicação e os acadêmicos da área de saúde. Rev Cienc Saude Coletiva. 2010;15(5):2533-2538.

14. Silva JD, Gomes AL, Oliveira JD, Sasaki YA, Maia BTB, Abreu BM. Prevalência de automedicação e os fatores associados entre os usuários de um Centro de Saúde Universitário. Rev Bras Clin Med. 2013;11(1):27-30.

15. Ribeiro MI, Oliveira A, Silva H, Mendes M, Almeida M, Silva T. Prevalência da automedicação na população estudantil do Instituto Politécnico de Bragança. Rev Portug Saude Pub. 2010;28(1):41-48.

16. Jesus PRC [homepage da internet]. O Consumo desenfreado de medicamentos no Brasil e a responsabilidade da propaganda. 2012 [acesso em 07 abril 2015]. Disponível em: http://www2.metodista.br/unesco/

17. Silva MG, Lourenço EE. Uso indiscriminado de anti-inflamatórios em Goiânia - GO e Bela Vista GO. Rev Cient ITPAC. 2014;7(4):1-7.

18. Queiroz TP, Santos PLD, Esteves JC, Stellin GM, Shimuzi AS, Betoni Junior W, Vieira EH. Dipirona versus paracetamol no controle da dor pós-operatória. Rev Odontol UNESP. 2013;78-82.

19. Araujo MAR. Hepatotoxicidade associada à nimesulida. Rev Bras Farm. 2012;93(3):283-289.

20. Espinosa Bosch M. Determination of paracetamol: historical evolution. J Pharm Biomed Anal. 2006;42:291-321.

21. Conceição S, Morais DCM. Automedicação com antibióticos em estabelecimento farmacêutico do município de Mogi Mirim, SP, Brasil. Rev Foco. 2012;3(3):23-34.

22. Brasil. Agência Nacional de Vigilância Sanitária e Instituto de Defesa do Consumidor. Vigilância sanitária, alimentos, medicamentos, produtos e serviços de interesse a saúde - guia didático. Brasília; 2009. 